\title{
Progression of cardiac allograft vascular disease as assessed by serial intravascular ultrasound: correlation to immunological and non-immunological risk factors
}

\author{
K Pethig, V Klauss, B Heublein, H Mudra, A Westphal, C Weber, K Theisen, A Haverich
}

\begin{abstract}
Objective-To characterise the severity and progression of cardiac allograft vascular disease (CAVD) in a large patient cohort, and to evaluate possible immunological and nonimmunological risk factors for progression.

Design-A prospective observational study using intravascular ultrasound.

Setting-Two university hospitals.

Patients and main outcome measures-Changes in focal plaque, lumen, and total vessel area (worst site method) were assessed at baseline and after 12.1 (2.8) months (mean (SD)) of follow up in a cohort of 96 patients (79 male, 17 female; mean age 48.7 (9.6) years; time post-transplant 26.0 (32.4) months).

Results-Overall, the mean (SD) intimal index of worst sites increased by $6.7(8.8) \%$. The increase in the first 12 months was $7.5(9.4) \%$, v 5.9 (8.0)\% after the first year (NS). Analysing immunological and non-immunological risk factors (age, underlying disease, sex, donor age, immunosuppression, cytomegalovirus, rejection episodes, cholesterol), low density lipoprotein (LDL) cholesterol was found to be the most important predictor of severe progression (as defined by an increase in intimal index of $\geqslant 15 \%(p=0.01)$.

Conclusions-Progression of CAVD is characterised by a continuing increase in intimal hyperplasia, especially within the first year after heart transplantation. LDL cholesterol is an important predictor of major progression.

(Heart 2000;84:494-498)
\end{abstract}

Keywords: heart transplantation; cardiac allograft vasculopathy; intravascular ultrasound

Endothelial dysfunction ${ }^{12}$ and multifocal myointimal hyperplasia ${ }^{3-5}$ are the major characteristics of cardiac allograft vascular disease (CAVD). Several non-invasive and invasive approaches have been used to characterise this accelerated coronary syndrome. ${ }^{67}$ However, intravascular ultrasound has proved to be the most sensitive technique for evaluating the severity and progression of vascular disease in transplanted hearts. ${ }^{8}$ In addition to angiographic information, not only luminal dimensions but all major vascular structures, as lumen, plaque, and total vessel area, can be assessed and quantified by intravascular ultrasound..$^{9}$ Recent large scale cross sectional studies have provided important insights into the prevalence and distribution pattern of intimal hyperplasia, ${ }^{11}{ }^{12}$ both early and late after transplantation. ${ }^{13}{ }^{14}$ However, in the individual patient it is necessary not only to determine whether or not disease is present but also the rate at which disease is progressing. Few studies have focused on this important issue so far. ${ }^{15-17}$ Moreover, beyond descriptive aspects, the question of potential risk factors, identifying patients with a rapid progressive course, is of major clinical interest.

Different approaches have been suggested to quantify the severity of disease. One of thesethe "worst site" method-is based on selection of the most diseased vascular sites (defined as the maximal focal intimal index) per vessel segment. ${ }^{11218}$ The clinical relevance of this approach was confirmed by Mehra et al and Rickenbacher et $a l^{1920}$ and more recently by Klauss et $a l l^{21}$ who showed that the severity of these lesions was of prognostic relevance. Focusing on a more diffuse manifestation of intimal hyperplasia, averaging procedures using either multiple randomly selected $\operatorname{sites}^{22}$ or a volumetric approach ${ }^{23}$ have been applied. Although these may be more suitable for quantifying the total plaque burden, the clinical relevance of these methods has not been demonstrated so far.

Our aim in this serial intravascular ultrasound study was: first, to characterise the pattern of progression of allograft vasculopathy in the most diseased sites in each vessel; second, to compare progression defined by intravascular ultrasound and by angiography; and third, to analyse possible donor, recipient, and graft related risk factors predicting a rapidly progressive course.

Using identical investigation protocols, this study was performed in two centres, allowing us to analyse data from 96 heart transplant recipients, each of whom had at least two intravascular ultrasound studies over a 12 month period.

\section{Methods}

PATIENTS

After obtaining written informed consent, 96 consecutive heart transplant recipients (centre 1 (Hannover) 46; centre 2 (Munich) 50; 79 
Table 1 Mean plaque, lumen, and vessel area at baseline and follow up examination

\begin{tabular}{|c|c|c|c|c|c|c|c|c|c|}
\hline & \multicolumn{3}{|c|}{ Overall $(n=96)$} & \multicolumn{3}{|c|}{$\leqslant 1$ year $(n=51)$} & \multicolumn{3}{|c|}{$>1$ year $(n=45)$} \\
\hline & Baseline & Follow up & p Value & Baseline & Follow up & p Value & Baseline & Follow up & $p$ Value \\
\hline Plaque area $\left(\mathrm{mm}^{2}\right)$ & $2.9(2.5)$ & $4.2(2.7)$ & $\star \star \star$ & $2.0(1.9)$ & $3.3(2.3)$ & $\star \star \star$ & $3.9(2.7)$ & $5.2(2.9)$ & $\star \star \star$ \\
\hline Lumen area $\left(\mathrm{mm}^{2}\right)$ & $10.7(3.7)$ & $10.3(3.4)$ & NS & $11.8(3.7)$ & $10.7(3.6)$ & NS & $9.4(3.2)$ & $9.9(3.1)$ & NS \\
\hline Vessel area $\left(\mathrm{mm}^{2}\right)$ & $13.6(4.1)$ & $14.5(4.2)$ & $\star \star$ & $13.8(10.6)$ & $13.9(4.4)$ & NS & $13.3(4.2)$ & $15.1(3.8)$ & $\star \star \star$ \\
\hline Intima index $(\%)$ & $20.2(15.4)$ & $26.8(15.5)$ & $\star \star \star$ & $13.8(4.1)$ & $21.2(12.7)$ & $\star \star \star$ & $27.4(16.9)$ & $33.2(16.0)$ & $\star \star \star \star$ \\
\hline
\end{tabular}

${ }^{\star \star} \mathrm{p}<0.005 ;{ }^{\star \star \star} \mathrm{p}<0.001 ; \mathrm{NS}$, not significant.

Table 2 Progression of allograft vascular disease over time

\begin{tabular}{lllll}
\hline Change in & Overall $(n=96)$ & $\leqslant 1$ year $(n=51)$ & $>1$ year $(n=45)$ & $p$ Value \\
\hline Plaque area $\left(\mathrm{mm}^{2}\right)$ & $1.3(1.7)$ & $1.3(1.9)$ & $1.3(1.4)$ & 0.87 \\
Lumen area $\left(\mathrm{mm}^{2}\right)$ & $-0.4(2.6)$ & $-1.1(2.8)$ & $0.4(2.1)$ & 0.003 \\
Vessel area $\left(\mathrm{mm}^{2}\right)$ & $0.9(3.0)$ & $0.2(3.1)$ & $1.7(2.7)$ & 0.009 \\
Intima index $(\%)$ & $6.7(8.8)$ & $7.5(9.4)$ & $5.9(8.0)$ & 0.35 \\
\hline
\end{tabular}

men and 17 women; mean (SD) age at transplantation 48.7 (9.6) years; time after heart transplantation 26.0 (32.4) months, range $0.3-116.4$ months) underwent routine surveillance heart catheterisation including a baseline intravascular ultrasound study. Follow up studies, using an identical protocol, were performed 12.1 (2.8) months later.

Immunosuppression was applied in the form of cyclosporin A, azathioprine, and prednisolone in 67 patients, cyclosporin $\mathrm{A}$ and prednisolone in 15, tacrolimus, azathioprine, and prednisolone in 12, and tacrolimus and prednisolone in two. Methotrexate was given instead of azathioprine in one patient and mycophenolate mofetil in another. Application of vasoactive drugs (angiotensin converting enzyme inhibitors, calcium channel blockers, nitrates) was discontinued 12 hours before the examination.

\section{INTRAVASCULAR ULTRASOUND STUDY}

Intravascular ultrasound was intended in the two major branches of the left coronary artery (left anterior descending and circumflex). Before intravascular placement of a 0.014 inch $(0.36 \mathrm{~mm})$ coronary guide wire, $10000 \mathrm{IU}$ of heparin and 100-200 $\mu \mathrm{g}$ of intracoronary glyceryl trinitrate were given into the target vessel. Following placement of the intravascular ultrasound catheter (monorail 4.3 French, $30 \mathrm{MHz}$, Sonicath, Boston Scientific, Andover, Massachusetts, USA $(n=45)$, or 2.9 French CVIS, CVIS Inc, Sunnyvale, California, USA $(n=51))$ under fluoroscopic guidance in the periphery of the coronary artery, avoiding lumen diameters of less than $2.0 \mathrm{~mm}$, the quality of the intravascular ultrasound images obtained was optimised using standardised gain settings. During a subsequent pull back manoeuvre ( $1 \mathrm{~mm} / \mathrm{s}$ using a motorised device), images were recorded on super VHS videotape for further analysis.

For follow up studies, an identical protocol using the same intravascular ultrasound system and settings was used.

ANGIOGRAPHIC ANALYSIS

Analysis of coronary angiograms was performed by two independent investigators (multiple projections, identical angulations during baseline and follow up examination). Based on visual estimation, the severity of
CAVD was graded using a standardised scale. To allow for comparison with data from intravascular ultrasound examinations, only vessels that were also assessed by intravascular ultrasound were used for grading. Progression of disease was defined as an increase of at least one step in scale.

\section{ULTRASOUND ANALYSIS}

Image analysis was performed off-line from the videotape using a conventional image analysis system (Tape Measure, Indec Systems, San Francisco, California, USA). After reviewing the complete imaging sequence, the analysed part of each vessel was divided into three segments: proximal, mid-vascular, and distal. The vascular site within each third of the vessel that had the highest intimal index was chosen for further analysis (worst site approach). "Lumen" was defined as the area within the intimal border, "total vessel" as the area within the media/adventitia boundary (characterised by the external border of the echolucent zone), and "plaque" as the space between vessel area and lumen area. Intimal index was calculated as plaque area divided by vessel area multiplied by 100 . The mean plaque area, mean vessel area, mean lumen area, and mean intimal index per patient were defined as mean values of all segments analysed. Identical protocols were applied at baseline and at the follow up examination. Our primary intention was to identify worst sites in each vessel segment; in many cases but not all, these reflected identical vascular sites. An increase in intimal index of more than $15 \%$ as compared with the baseline examination was regarded as representing severe progression.

\section{STATISTICAL ANALYSIS}

Statistical analysis was performed using a computer assisted software package (SPSS, version 6.1.3). Continuous data are presented as mean (SD). A probability value $\mathrm{p}<0.05$ was considered significant.

The Student $t$ test was used to compare for differences in intimal index, plaque, lumen, and vessel area within the first year ("early period") and beyond the first year ("late period") after transplantation.

Bivariate assessment for statistical differences in immunological and nonimmunological risk factors between patients with and without severe progression was done using the $\chi^{2}$ test (non-continuous variables) and the $t$ test (continuous variables). In addition a multivariate analysis was performed by logistic regression (backward, LR). 


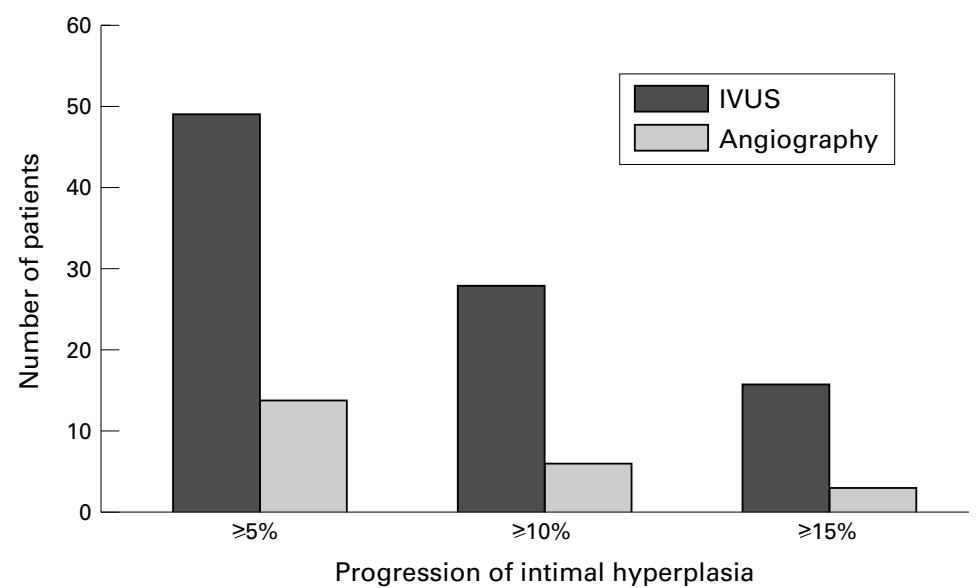

Figure 1 Progression of intimal hyperplasia by intravascular ultrasound in comparison with coronary angiography findings. Note the low sensitivity of angiography in detecting an increase in intimal index over a wide range.

\section{Results}

Serial intravascular ultrasound studies were performed in 159 vessels, 1.7 (0.5) vessels/ patient. Two vessels (left anterior descending and circumflex) were examined in 63 patients and one vessel in 33 (left anterior descending in 29; circumflex in four). In all, 475 vascular sites (4.9 (1.4) sites per patient) were evaluated.

PLAQUE AREA

A uniform and significant increase by $1.3 \mathrm{~mm}$ $(p<0.001)$ was observed in the total patient cohort as well as in the subgroups $\leqslant 1$ year and $>1$ year after transplantation (tables 1 and 2).

LUMEN AREA

Luminal area did not change significantly between baseline and follow up examination in the cohort as a whole. However, when the patients were broken down by time after transplantation, within the first year after their transplant there was a decrease in mean lumen area $(-1.1(2.8) \mathrm{mm})$, while after the first year there was a small but significant increase in lumen area $(+0.4(2.1) \mathrm{mm} ; \mathrm{p}=0.003)$ (tables 1 and 2 .
VESSEL AREA

During follow up an overall increase in total vessel area was observed in all patients (from $13.6(4.1) \mathrm{mm}$ to $14.5(4.2) \mathrm{mm}, \mathrm{p}<0.005)$ (table 1). Analysing the subgroups early and late after transplantation, the increase in vessel area was mainly a late feature, with an increase of $1.7(2.7) \mathrm{mm}$ after the first year compared with 0.2 (3.1) $\mathrm{mm}$ within the first 12 months $(\mathrm{p}<0.009)$ (table 2, fig 1$)$.

\section{INTIMAL INDEX}

A constant and significant increase in mean intimal index was observed throughout the entire observation period. There were no statistical differences between the early and late periods.

\section{RISK FACTOR ANALYSIS}

To identify a subgroup with severe progression of disease, we chose for further risk factor analysis patients with an increase in intimal index of $\geqslant 15 \%$ ( $n=16,16.6 \%$ of the total patient cohort, 11 of 51 patients within the first year, five of 45 patients beyond the first year). Comparing this subgroup with patients with a smaller increase in intimal index $(\leqslant 5 \%)$, we assessed several immunological and nonimmunological risk factors (age, underlying disease, sex, donor age, type of immunosuppression, cytomegalovirus infection, rejection episodes, cholesterol, high density lipoprotein (HDL) cholesterol, low density lipoprotein (LDL) cholesterol). Both bivariate and multivariable analysis (logistic regression) identified LDL cholesterol $(\mathrm{p}=0.01)$ as the only factor associated with severe progression (table 3 ).

\section{ANGIOGRAPHIC PROGRESSION}

Using a semiquantitative score (grade 0 to grade 4 , table 4 ), angiography revealed progression of disease by at least one grade in 22 patients ( 10 of 51 within the first year and 12 of 45 beyond the first year after transplantation). However, only 14 of 49 patients with an increase in intimal index of $\geqslant 5 \%$, six of 28 with an increase of $\geqslant 10 \%$, and three of 16

Table 3 Risk factors for progression of intimal index $(\geqslant 15 \% v \leqslant 5 \%)$

\begin{tabular}{lllllll}
\hline & & Overall $(n=96)$ & $\begin{array}{l}\text { Progression } \leqslant 5 \% \\
(n=46)\end{array}$ & $\begin{array}{l}\text { Progression } \geqslant 15 \% \\
(n=16)\end{array}$ & $\begin{array}{l}p \text { Value } \\
\text { (bivariate) }\end{array}$ & $\begin{array}{c}p \text { Value } \\
(\text { multivariate })\end{array}$ \\
\hline Age & years & 48.7 & $49.4(8.2)$ & $50.0(9.4)$ & 0.82 & 0.25 \\
Underlying disease & CAD/other & $34 / 62$ & $19 / 27$ & $6 / 10$ & 0.62 & 0.3 \\
Sex & male/female & $79 / 17$ & $36 / 10$ & $14 / 2$ & 0.44 & 0.17 \\
Donor age & years & $33.6(11.9)$ & $33.4(12.9)$ & $30.5(11.1)$ & 0.40 & 0.57 \\
Immunosuppression & CyA/Tac & $81 / 15$ & $40 / 6$ & $13 / 3$ & 0.72 & 0.81 \\
CMV disease & pos/neg & $0.15(0.51)$ & $0.15(0.47)$ & $0.25(0.78)$ & 0.63 & 0.49 \\
Rejection episodes & number & $1.3(1.7)$ & $1.1(1.5)$ & $1.3(1.4)$ & 0.82 & 0.23 \\
Cholesterol & mmol/1 & $6.27(1.17)$ & $6.08(1.42)$ & $6.66(1.42)$ & 0.17 & 0.89 \\
HDL cholesterol & mmol/1 & $1.39(0.48)$ & $1.42(0.45)$ & $1.28(0.42)$ & 0.29 & 0.17 \\
LDL cholesterol & mmol/1 & $3.94(1.26)$ & $3.71(1.29)$ & $4.57(0.99)$ & 0.01 & 0.02
\end{tabular}

CAD, coronary artery disease; CMV, cytomegalovirus; CyA, cyclosporin A; Tac, tacrolimus; HDL, high density lipoprotein; LDL, low density lipoprotein.

Table 4 Angiographic progression of disease

\begin{tabular}{lllllll}
\hline & Total & Angio grade 0 & Angio grade 1 & Angio grade 2 & Angio grade 3 & Angio grade 4 \\
\hline Baseline study (n (\%)) & 96 & $69(71.9 \%)$ & $11(11.5 \%)$ & $8(8.3 \%)$ & $6(6.3 \%)$ & $2(2.1 \%)$ \\
Follow up study (n (\%)) & 96 & $59(61.5 \%)$ & $13(13.5 \%)$ & $8(8.3 \%)$ & $13(13.5 \%)$ & $3(3.1 \%)$
\end{tabular}

Grade 0, normal angiogram; grade 1, wall irregularities with luminal obstruction $<30 \%$; grade 2, luminal obstruction $30-50 \%$; grade 3 , luminal obstruction $\geqslant 50 \%$; grade 4 , vessel occlusion. 
with an increase of $\geqslant 15 \%$ were identified by coronary angiographic progression (fig 1).

\section{Discussion}

Since the introduction of intravascular ultrasound into clinical practice, several cross sectional studies have been performed to define the severity and distribution pattern of cardiac allograft vascular disease. ${ }^{11}{ }^{12}$ However, little is known about the characteristics of progression-in our opinion essential information not only in relation to its scientific aspects but also for the clinical management of the patients. Using intravascular ultrasound, our aim in this study was to define the natural course of progression, to identify recipients at risk, and to assess possible risk factors predicting a rapidly progressive course.

Overall, CAVD was confirmed to be an accelerated coronary syndrome. Using the worst site approach, mean intimal index could be shown to increase by $6.7(8.8) \%$ in 12 months, and the increase was particularly pronounced within the first year after transplantation (7.5 (9.4)\%, compared with 5.9 (8.0)\% after the first year). Bivariate and multivariate analysis showed that LDL cholesterol was the most important and only significant risk indicator for severe progression, defined as an increase in the intimal index $\geqslant 15 \%$.

PROGRESSION OF DISEASE: ANGIOGRAPHY OR INTRAVASCULAR ULTRASOUND

Intravascular ultrasound can be regarded as the optimal imaging procedure for characterising intimal hyperplasia and luminal loss in CAVD,${ }^{25}$ while angiographic methods have been shown to be insensitive. ${ }^{18}$ This was confirmed in the present study. Less than 30\% of patients found to have an increasing intimal index on intravascular ultrasound examination had detectable changes in their coronary angiography. This supports the important role of intravascular ultrasound as a diagnostic tool in any therapeutic approach.

To define the natural course of progression, we considered the following issues to be of special interest. First, when does progression occur? Second, what is the mean progression rate over time? And third, what are the vascular structures involved in progression?

Histopathological studies and data from a multicentre intravascular ultrasound trial have shown that the largest increase in intimal index occurs within the first year after heart transplantation. ${ }^{1526}$ Those results were confirmed by our present study. However, long term angiographic follow up has shown that progression of disease continues after the first year. ${ }^{27}$ Thus at a mean (SD) of 4.6 (2.3) years after transplantation, there was only a slightly smaller increase in intimal index than within the first year (5.9 (8.0)\% $v 7.5(9.4) \%$, NS). This is of importance for long term clinical management. Diagnostic and therapeutic procedures should therefore not be limited to the early postoperative period, but should be continued during later follow up as well. Although therapeutic options are limited, lipid lowering treatment might be effective in selected patients. ${ }^{28}{ }^{29}$ Furthermore, encouraging results with interventional procedures such as stent implantation $^{30}$ suggest that such techniques may become part of the routine approach in cases of critical luminal obstruction.

Apart from the quantification of plaque area, there is increasing interest in the question of coronary artery remodelling. ${ }^{31}$ Compensatory enlargement as well as localised reduction in total vessel area can be demonstrated in CAVD. ${ }^{32}{ }^{33}$ Our findings in the present study support the presence of a remodelling process. While plaque area was found to increase constantly over the entire observation period, a significant loss in lumen area was observed only in the first year after transplantation. Despite a similar increase in plaque mass, at later times after transplantation there was compensatory enlargement of total vessel area, resulting in a slight increase (instead of a decrease) in luminal dimensions. Therefore an increase in plaque area might represent only one aspect of coronary pathology.

\section{RISK FACTORS}

In view of the likely presence of remodelling processes, we chose the intimal index as a combined measure, representing plaque as well as vessel area, to characterise progression. Our analysis shows that progression of CAVD is highly variable between individuals. While the majority of patients presented with a moderate increase in intimal index, 16 were found to have severe progression ( $\geqslant 15 \%$ increase in intimal index). Assuming that these differences in progression may be influenced by patient, graft, or environment related factors, we analysed multiple immunological and nonimmunological variables in patients presenting with severe progression (11 within the first year and five beyond the first year after transplantation). Using both bivariate and multivariate analysis, LDL cholesterol (4.57 (0.99) mmol/1 v $3.71(1.29) \mathrm{mmol} / \mathrm{l})$ was found to be significantly increased in the progression group. These findings correspond to previously published results from cross sectional and interventional studies, ${ }^{28} 2934$ showing raised cholesterol concentrations to be one of the most important factors associated with abnormal intimal thickening. Intracellular and extracellular lipid deposition and the induction of myointimal hyperplasia are thought to be the most probable mechanisms in pathogenesis. ${ }^{35}$ These results suggest that effective lipid lowering treatment should be offered to these patients, especially those with progressive intimal hyperplasia.

In contrast to previous findings, other immunological and non-immunological risk factors (such as rejection episodes, immunosuppression, cytomegalovirus infection, donor age, and $\operatorname{sex}^{36-38}$ ) were not found to correlate with severe progression of disease in the present study. Because of the comparatively short observation period, our current data do not rule out the possibility that these factors may play a part. However, they would appear to be of minor importance in the setting analysed here. 
LIMITATIONS

As discussed above, progression of CAVD is a complex and continuing process. This study represents a first approach to characterising its progression in a large patient cohort. A definite limitation is the follow up period of only 12 months, which precludes any speculation about longer periods of observation. A second limitation is that the analysis focused on the worst sites of each vessel segment in identifying the maximum severity of disease. However, in view of the possible remodelling process, this procedure may not be optimal. Further studies with plaque volumetry in defined segments may improve the comparability of the measurements. Finally, in order to collect an acceptable sample size, this study was carried out in two centres. Although the therapeutic and diagnostic protocols were nearly identical and the patient characteristics and the pattern of progression did not differ significantly between the two centres, minor differences in management cannot be ruled out.

CONCLUSIONS

Serial intravascular ultrasound examinations showed that progression of cardiac allograft vasculopathy was characterised by a continuing increase in intimal hyperplasia, which was most pronounced within the first year after heart transplantation. Using the worst site approach, the degree of luminal narrowing was shown to be influenced not only by an increase in plaque area but also by coronary artery remodelling processes. There was a large degree of interindividual variability in progression. LDL cholesterol was shown to be an important predictor of severe progression.

1 Anderson TJ, Meredith IT, Uehata A, et al. Functional significance of intimal thickening as detected by intravascular ultrasound early and late after cardiac transplantation. Cirultrasound early and late after

2 Klauss V, Ackermann K, Hennecke K-H, et al. Epicardial intimal thickening in transplant coronary artery disease intimal thickening in transplant coronary artery disease and resistance vessel respon

3 Hosenpud JD, Shipley GD, Wagner CR. Cardiac allograft vasculopathy: current concepts, recent developments, and future directions. F Heart Lung Transplant 1992;11:9-23.

4 Schmid C, Kerber S, Baba HA, et al. Graft vascular disease after heart transplantation. Eur Heart f 1997;18:554-9.

5 Weis M, von Scheidt W. Cardiac allograft vasculopathy. A review. Circulation 1997;96:2069-77.

6 Smart FW, Ballantyne CM, Cocanougher B, et al. Insensitivity of noninvasive tests to detect coronary artery vasculopathy after heart transplantation. Am 7 Cardiol 1991;67:243-7.

7 Gao AZ, Alderman EL, Schroeder JS, et al. Accelerated coronary vascular disease in the heart transplant patient: coronary arteriographic findings. $\mathcal{f}$ Am Coll Cardiol 1988;12: 334-40.

8 Waller BF, Pinkerton CA, Slack JD. Intravascular ultrasound: a histological study of vessels during life. The new "gold standard" for vascular imaging. Circulation $1992 ; 85 \cdot 2305-10$

9 Nishimura RA, Edwards WD, Warnes CA. Intravascular ultrasound imaging: in vitro validation and pathologic correlation. $\mathcal{A}$ Am Coll Cardiol 1990;16:145-54.

10 Nissen SE, Gurley JC, Grines CL, et al. Intravascular ultrasound assessment of lumen size and wall morphology in normal subjects and patients with coronary heart disease. Circulation 1991;84:1087-99.

11 Chenzbraun A, Pinto F, Alderman E, et al. Distribution and morphologic features of coronary artery disease in cardiac allografts: an intracoronary ultrasound study. $\mathcal{f} \mathrm{Am}$ Soc Echocardiogr 1995;8:1-8.

12 Klauss V, Mudra H, Uberfuhr P, et al. Intraindividual variability of cardiac allograft vasculopathy as assessed by intravascular ultrasound. Am f Cardiol 1995;76:463-6.
13 Pinto FJ, Chenzbraun A, Botas J, et al. Feasibility of serial intracoronary ultrasound imaging for assessment of
progression of intimal proliferation in cardiac transplant progression of intimal proliferation in
recipients. Circulation 1994;90:2348-55.

14 Rickenbacher PR, Pinto FJ, Chenzbraun A, et al. Incidence and severity of transplant coronary artery disease early and up to 15 years after transplantation as detected by intravascular ultrasound. F Am Coll Cardiol 1995;25:171-7.

15 Yeung AC, Davis SF, Hauptmann PJ, et al. Incidence and progression of transplant coronary artery disease over 1 year: results of a multicenter trial with use of intravascular ultrasound. F Heart Lung Transplant 1995;14:S215-20.

16 Mehra MR, Ventura HO, Chambers R, et al. Predictive model to assess risk for cardiac allograft vasculopathy: an
intravascular ultrasound study. 7 Am Coll Cardiol 1995;26: 1537-44.

17 Kapadia SR, Nissen SE, Ziada KM, et al. Development of transplantation vasculopathy and progression of donorransmitted atherosclerosis. Comparison by serial intravascular ultrasound imaging. Circulation 1998;98:2672-8.

18 St Goar FG, Pinto FJ, Alderman EL, et al. Intracoronary ultrasound in cardiac transplant recipients. In vivo evidence of "angiographically silent" intimal thickening [see comments]. Circulation 1992;85:979-87.

19 Mehra MR, Ventura HO, Stapleton DD, et al. Presence of severe intimal thickening by intravascular ultrasonography predicts cardiac events in cardiac allograft vasculopathy. $\mathcal{F}$ Heart Lung Transplant 1995;14:632-9.

20 Rickenbacher PR, Pinto FJ, Lewis NP, et al. Prognostic importance of intimal thickness as measured by intracoronary ultrasound after cardiac transplantation. Circulation 1995;92:3445-52.

21 Klauss V, Pethig K, Kalies $\mathrm{H}$, et al. Prognostic impact of intravascular ultrasound after heart transplantation: a multivariable analysis in a large patient cohort [abstract]. $\mathcal{F} \mathrm{Am}$ Coll Cardiol 1999;33:218A.

22 Johnson JA, Kobashigawa JA. Quantitative analysis of transplant coronary artery disease with use of intracoronary ultrasound. F Heart Lung Transplant 1995;14:S198-201.

23 von Birgelen C, Slager CJ, Di Mario C, et al. Volumetric intracoronary ultrasound: a new maximum confidence approach for the quantitative assessment of progression-
regression of atherosclerosis? Atherosclerosis 1995;118: S103-13.

24 Pethig K, Heublein B, Meliss RR, et al. Volumetric remodeling of the proximal left coronary artery-early versus late after heart transplantation. $\mathcal{F}$ Am Coll Cardiol 1999;34:197203.

25 St Goar FG, Pinto FJ, Alderman EL, et al. Intravascular ultrasound imaging of angiographically normal coronary arteries: an in vivo comparison with quantitative angiography. F Am Coll Cardiol 1991;18:952-8.

26 Johnson DE, Gao SZ, Schroeder JS, et al. The spectrum of coronary artery pathologic findings in human cardiac allocoronary artery pathologic findings in humar

27 Pethig K, Besser K, Heublein B, et al. Allograft vasculopathy after heart transplantation: Impact of time related course, severity, and progression on prognosis during long-term follow-up. Z Kardiol 1999;88:498-506.

28 Wenke K, Meiser B, Thiery J, et al. Simvastatin reduces graft vessel disease and mortality after heart transplantation. Circulation 1997;96:1398-402.

29 Park JW, Merz M, Braun P. Regression of transplant coronary artery disease during chronic low-density lipoproteinapheresis. F Heart Lung Transplant 1997;16:290-7.

30 Heublein B, Pethig K, Maass C, et al. Coronary stenting in cardiac allograft vascular disease. Am Heart $\mathcal{F}$ 1997;134: $930-8$

31 Gibbons GH. The pathogenesis of graft vascular disease: implications of vascular remodeling. F Heart Lung Transplant 1995;14:S149-58.

$32 \mathrm{Lim}$ TT, Liang D, Botas J, et al. Role of compensatory enlargement and shrinkage in transplant coronary artery disease. Serial intravascular ultrasound study. Circulation 1997;95:855-9.

33 Pethig K, Heublein B, Wahlers T. Impact of plaque burden on compensatory enlargement of coronary arteries in cardiac allograft vasculopathy. Am $\mathcal{f}$ Cardiol 1997;79:8992.

34 Kobashigawa JA, Katznelson S, Laks H, et al. Effect of pravastatin on outcomes after cardiac transplantation. $N$ Engl 7 Med 1995;333:621-7.

35 Shi C, Lee W-S, Russel M, et al. Hypercholesterolemia exacerbates transplant arteriosclerosis via increased neointimal smooth muscle cell accumulation. Circulation 1997;96:2722-8.

36 Escobar A, Ventura HO, Stapleton DD, et al. Cardiac allograft vasculopathy assessed by intravascular ultrasonography and nonimmunologic risk factors. $\mathrm{Am} \mathcal{F}$ Cardiol 1994;74:1042-6.

37 Costanzo Nordin MR. Cardiac allograft vasculopathy: relationship with acute cellular rejection and histocompatibility. F Heart Lung Transplant 1992;11:S90-103.

38 Hornick P, Smith J, Pomerance A, et al. Influence of acute rejection episodes, HLA matching, and donor/recipient phenotype on the development of "early" transplantassociated coronary artery disease. Circulation 1997; 96(suppl II):II-148-53. 